\title{
Limit cycles bifurcating from the periodic annulus of the weight-homogeneous polynomial centers of weight-degree 2
}

\author{
J. Llibre ${ }^{a}$, B.D. Lopes ${ }^{\mathrm{a}, \mathrm{b}}$, J.R. de Moraes ${ }^{\mathrm{c}, *}$ \\ a Departament de Matemàtiques, Universitat Autònoma de Barcelona, Bellaterra, Barcelona 08193, Catalonia, Spain

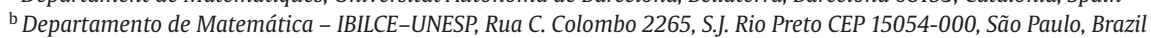 \\ ' Curso de Matemática - UEMS, Rodovia Dourados-Itaum Km 12, Dourados, Mato Grosso do Sul CEP 79804-970, Brazil
}

\section{A R T I C L E I N F O}

\section{MSC:}

$34 \mathrm{C07}$

$34 \mathrm{C} 23$

$34 \mathrm{C} 25$

$34 \mathrm{C} 29$

$37 \mathrm{C} 10$

$37 \mathrm{C} 27$

$37 \mathrm{G} 15$

Keywords:

Polynomial vector field

Limit cycle

Averaging method

Weight-homogeneous differential system

\begin{abstract}
A B S T R A C T
We obtain an explicit polynomial whose simple positive real roots provide the limit cycles which bifurcate from the periodic orbits of a family of cubic polynomial differential centers when it is perturbed inside the class of all cubic polynomial differential systems. The family considered is the unique family of weight-homogeneous polynomial differential systems of weight-degree 2 with a center. The computations has been done with the help of the algebraic manipulator Mathematica.
\end{abstract}

(c) 2015 Elsevier Inc. All rights reserved.

\section{Introduction and statement of the main results}

The study of the number of limit cycles of a polynomial differential system is mainly motivated by the16th Hilbert's problem stated in 1900. See [9] and [15] for more details.

Actually one of the main goals in the qualitative theory of real planar polynomial differential systems is the determination of their limit cycles. One of the ways to produce limit cycles is perturbing a polynomial differential system which has a center and to study the number of limit cycles which can bifurcate from the periodic orbits of the center, up to first order in the small parameter of the perturbation, see for instance $[1,3,8,10,14]$. This problem is called for some authors the weak Hilbert's problem.

There are many methods to study the maximum number of limit cycles that bifurcate from the periodic annulus of a center, i.e. to study the weak Hilbert's problem. Most of them are based on the Poincaré return map, the Poincaré-Melnikov integrals, the Abelian integrals, and the averaging theory. It is well known that in the plane the last three methods are essentially equivalent. The weak Hilbert's problem has been studied by many authors, see for instance the second part of the book [6] and the hundreds of references quoted there. See also [12].

Here we consider polynomial differential systems given by

$$
\begin{aligned}
& \dot{x}=P(x, y), \\
& \dot{y}=Q(x, y),
\end{aligned}
$$

\footnotetext{
* Corresponding author. Tel.: +55 6730327078.

E-mail addresses: jlibre@mat.uab.cat (J. Llibre), brunodomicianolopes@gmail.com (B.D. Lopes), jaime@uems.br, jrezmoraes@gmail.com (J.R. de Moraes).
} 


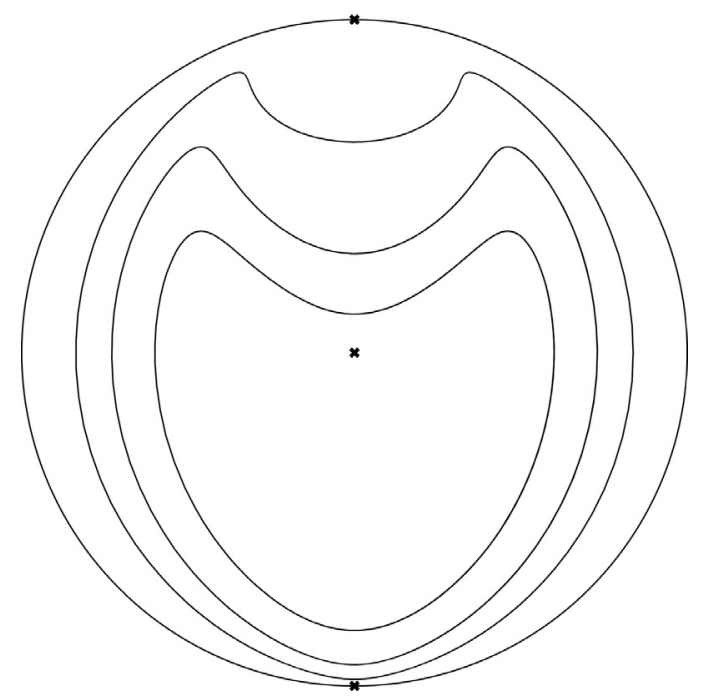

Fig. 1. Phase portrait of the polynomial differential system (2) in the Poincaré disc.

where $P$ and $Q$ are polynomials with real coefficients, the degree of the system is the maximum of the degrees of the polynomials $P$ and $Q$.

System (1) is called weight-homogeneous if there exist $\left(s_{1}, s_{2}\right) \in \mathbb{N}^{2}$ and $d \in \mathbb{N}$ such that for any $\lambda \in \mathbb{R}^{+}=\{\lambda \in \mathbb{R}: \lambda>0\}$ we have

$$
P\left(\lambda^{s_{1}} x, \lambda^{s_{2}} y\right)=\lambda^{s_{1}-1+d} P(x, y), \quad Q\left(\lambda^{s_{1}} x, \lambda^{s_{2}} y\right)=\lambda^{s_{2}-1+d} Q(x, y) .
$$

The vector $\left(s_{1}, s_{2}\right)$ is called the weight-exponent of system (1) and $d$ is called weight-degree with respect to the weight-exponent $\left(s_{1}, s_{2}\right)$.

There are few works trying to study the weak Hilbert's problem for weight-homogeneous polynomial differential systems. Our main goal is to solve the weak Hilbert's problem for the weight-homogeneous polynomial differential systems of weightdegree 2.

In [11] the authors classified all centers of a planar weight-homogeneous polynomial differential systems up to weight-degree 4. In particular they proved that the unique family of weight-homogeneous polynomial differential systems with a center with weight-degree 2 is

$$
\begin{aligned}
& \dot{x}=a_{20} x^{2}+a_{01} y=P(x, y), \\
& \dot{y}=b_{30} x^{3}+b_{11} x y=Q(x, y),
\end{aligned}
$$

with

$$
\left(b_{11}-2 a_{20}\right)^{2}+8 a_{01} b_{30}=-a^{2}<0 .
$$

The weight-exponent of this family is $\left(s_{1}, s_{2}\right)=(1,2)$.

The polynomial differential system (2) has a global center at the origin of coordinates, and its global phase portrait in the Poincaré disc is given in Fig. 1. For more details in order to study the global phase portrait of a polynomial differential system in the Poincaré disc see Chapter 5 of [7].

The main goal of this paper is to provide an explicit polynomial whose real positive simple zeros gives the exact number of limit cycles which bifurcate, at first order in the perturbation parameter, from the periodic orbits of the center of the weight-homogeneous polynomial differential system (2).

More precisely we consider the polynomial differential system

$$
\begin{aligned}
& \dot{x}=a_{20} x^{2}+a_{01} y+\varepsilon p(x, y), \\
& \dot{y}=b_{30} x^{3}+b_{11} x y+\varepsilon q(x, y),
\end{aligned}
$$

where

$$
\begin{aligned}
p(x, y)= & c_{00}+c_{10} x+c_{01} y+c_{20} x^{2}+c_{11} x y+c_{02} y^{2}+c_{30} x^{3}+c_{21} x^{2} y \\
& +c_{12} x y^{2}+c_{03} y^{3}, \\
q(x, y)= & d_{00}+d_{10} x+d_{01} y+d_{20} x^{2}+d_{11} x y+d_{02} y^{2}+d_{30} x^{3}+d_{21} x^{2} y \\
& +d_{12} x y^{2}+d_{03} y^{3},
\end{aligned}
$$

and $\varepsilon$ is a small parameter. 
Consider the following function

$$
g_{1}(\theta)=a^{2} \cos ^{4} \theta+\left(4 a_{01} \sin \theta+\left(2 a_{20}-b_{11}\right) \cos ^{2} \theta\right)^{2} .
$$

In what follows we state our main result where the functions $f_{i}(\theta)$, for $i=0,1, \ldots, 7, W(\theta)$ and $k(\theta)$ are given in Section 3 . We do not provide these functions here due to their length.

Theorem 1. Let $|\varepsilon|>0$ be a sufficiently small parameter and let $r_{0}^{*}$ be a positive simple root of the polynomial

$$
r_{0}^{2} \mathcal{F}\left(r_{0}\right)=\frac{1}{2 \pi} \sum_{k=0}^{3} r_{0}^{2 k} \int_{0}^{2 \pi} A_{2 k}(\theta) d \theta
$$

where

$$
A_{i}(\theta)=\frac{W(\theta) f_{i}(\theta) k(\theta)^{i-3}}{g_{1}(\theta)^{2}} .
$$

Then the following two statement hold.

(a) The perturbed systems (3) have a limit cycle bifurcating from the periodic orbit $r\left(\theta, r_{0}^{*}\right)=k(\theta) r_{0}^{*}$ of the period annulus of the center (2) using the averaging theory of first order.

(b) The polynomial (4) can have 0,1,2 or 3 positive simple real roots depending on the perturbation of system (2).

Statement (a) of Theorem 1 is proved in Section 3, and statement (b) in Section 4.

Note that from Theorem 1 it follows that using averaging theory of first order at most 3 limit cycles can bifurcate from the periodic solutions of the center (2).

\section{Preliminaries}

In this section we give some well known results that we shall need for proving Theorem 1 .

Consider a system given by

$$
\dot{\mathrm{x}}=F_{0}(t, \mathrm{x})+\varepsilon F_{1}(t, \mathrm{x})+\mathcal{O}\left(\varepsilon^{2}\right),
$$

where $\varepsilon \neq 0$ is sufficiently small and the functions $F_{0}, F_{1}: \mathbb{R} \times \Omega \rightarrow \mathbb{R}^{n}$ and $F_{2}: \mathbb{R} \times \Omega \times\left(-\varepsilon_{0}, \varepsilon_{0}\right) \rightarrow \mathbb{R}^{n}$ are $\mathcal{C}^{2}$ functions, $T$-periodic in the first variable and $\Omega$ is an open subset of $\mathbb{R}^{n}$. We suppose that the unperturbed system

$$
\dot{\mathrm{x}}=F_{0}(t, \mathrm{x})
$$

has a submanifold of periodic solutions of dimension $n$.

Let $x(t, z, \varepsilon)$ be the solution of system $(6)$ such that $\mathrm{x}(0, \mathrm{z}, \varepsilon)=z$. The linearization of the unperturbed system along a periodic solution $\mathrm{x}(t, \mathrm{z}, 0)$ is

$$
\dot{\mathrm{y}}=D_{\mathrm{x}} F_{0}(t, \mathrm{x}(t, \mathrm{z}, 0)) \mathrm{y} .
$$

In what follows we denote by $M_{z}(t)$ the fundamental matrix of the linearized system $(7)$ such that $M_{z}(0)$ is the identity matrix.

We assume that there is an open set $U$ with $\mathrm{Cl}(U) \subset \Omega$ such that for each $z \in \mathrm{Cl}(U), \mathrm{x}(t, \mathrm{z}, 0)$ is $T$-periodic, where $\mathrm{x}(t, z, 0)$ denotes the solution of the unperturbed system (6). We denote by $\mathrm{Cl}(U)$ the closure of $U$. The set $\mathrm{Cl}(U)$ is isochronous for system (6), i.e. it is formed only by periodic orbits with period $T$.

The following result is a version of averaging theorem for studying the bifurcation of $T$-periodic solutions of system (5) from the periodic solutions $\mathrm{x}(t, z, 0)$ contained in $\mathrm{Cl}(U)$ of system $(6)$ when $|\varepsilon|>0$ is sufficiently small. See [4] for a proof. For more details on the averaging theory see [5] and the book [13].

Theorem 2 (Perturbations of an isochronous set). We assume that there exists an open and bounded set $U$ with $\mathrm{Cl}(U) \subset \Omega$ such that for each $\mathrm{z} \in \mathrm{Cl}(U)$, the solution $\mathrm{x}(r, \mathrm{z}, 0)$ is $T$-periodic. Consider the function $\mathcal{F}: \mathrm{Cl}(U) \rightarrow \mathbb{R}^{n}$

$$
\mathcal{F}(\mathrm{z})=\frac{1}{T} \int_{0}^{T} M_{\mathrm{z}}^{-1}(t) F_{1}(t, \mathrm{x}(t, \mathrm{z}, 0)) d t .
$$

Then the following statements hold.

(i) If there exists $\mathbf{a} \in U$ with $\mathcal{F}(\mathbf{a})=0$ and $\operatorname{det}((\partial \mathcal{F} / \partial \mathrm{z})(\mathbf{a})) \neq 0$ then there exists a T-periodic solution $\mathrm{x}(t, \varepsilon)$ of system $(5)$ such that $\mathrm{x}(0, \varepsilon) \rightarrow$ a when $\varepsilon \rightarrow 0$.

(ii) The kind of the stability of the periodic solution $\mathrm{x}(t, \varepsilon)$ is given by the eigenvalues of the Jacobian matrix $((\partial \mathcal{F} / \partial \mathrm{z})(\mathbf{a}))$.

The following result is the generalized Descartes Theorem about the number of zeros of a real polynomial. See [2] for a proof.

Theorem 3. Consider the real polynomial $p(x)=a_{i_{1}} x^{i_{1}}+a_{i_{2}} x^{i_{2}}+\cdots+a_{i_{r}} x^{i_{r}}$ with $0 \leq i_{1}<i_{2}<\cdots<i_{r}$ and $a_{i_{j}} \neq 0$ real constants for $j \in\{1,2, \ldots, r\}$. When $a_{i_{j}} a_{i_{j+1}}<0$, we say that $a_{i_{j}}$ and $a_{i_{j+1}}$ have a variation of sign. If the number of variations of signs is $m$, then $p(x)$ has at most $m$ positive real roots. Moreover, it is always possible to choose the coefficients of $p(x)$ in such a way that $p(x)$ has exactly $r-1$ positive real roots. 


\section{Proof of statement (a) of Theorem 1}

We write system (3) in the generalized polar coordinates $x=r \cos \theta, y=r^{2} \sin \theta$, and we obtain the differential equation

$$
\frac{d r}{d \theta}=F_{0}(r, \theta)+\varepsilon F_{1}(r, \theta)+\mathcal{O}\left(\varepsilon^{2}\right)
$$

in the standard form for applying the averaging theory of first order described in Section 2, where

$$
\begin{aligned}
F_{0}(r, \theta)= & \frac{h_{1}(\theta)}{g_{1}(\theta)} r, \\
F_{1}(r, \theta)= & \frac{16 a_{01}^{2}\left(\cos ^{2} \theta+2 \sin ^{2} \theta\right)}{r^{4} g_{1}(\theta)^{2}}\left(Q\left(r \cos \theta, r^{2} \sin \theta\right) p\left(r \cos \theta, r^{2} \sin \theta\right)\right. \\
& \left.-P\left(r \cos \theta, r^{2} \sin \theta\right) q\left(r \cos \theta, r^{2} \sin \theta\right)\right), \\
h_{1}(\theta)= & r \cos \theta\left(\operatorname { c o s } ^ { 2 } \theta \left(\left(a^{2}+\left(b_{11}-2 a_{20}\right)^{2}\right) \sin \theta\right.\right. \\
& \left.\left.-8 a_{01} a_{20}\right)-8 a_{01}\left(a_{01}+b_{11} \sin \theta\right) \sin \theta\right) .
\end{aligned}
$$

Note that the differential Eq. (9) satisfies the assumptions of Theorem 2. Consider $r\left(\theta, r_{0}\right)$ the periodic solution of the differential equation $d r / d \theta=r h_{1}(\theta) / g_{1}(\theta)$ such that $r\left(0, r_{0}\right)=r_{0}$. For solving this differential equation we take $z=\sin \theta$ in $g_{1}(\theta)$, and we obtain a polynomial of degree 4 in $z$ which can be factorized in the form

$$
g_{1}(z)=H_{1}\left(z-z_{1}\right)\left(z-z_{2}\right)\left(z-z_{3}\right)\left(z-z_{4}\right),
$$

where $H_{1}=a^{2}+\left(b_{11}-2 a_{20}\right)^{2}$, the coefficients of $g_{1}^{*}(z)=\left(z-z_{1}\right)\left(z-z_{2}\right)$ and $g_{2}^{*}(z)=\left(z-z_{3}\right)\left(z-z_{4}\right)$ are reals, and $z_{i}$ are the complex roots of $g_{1}$ for $i=1, \ldots, 4$ given by

$$
\begin{aligned}
& z_{1}=H_{3}-\sqrt{\frac{N_{1}}{H_{1}^{2}}}-2 H_{2}, \quad z_{2}=H_{3}-\sqrt{\frac{N_{2}}{H_{1}^{2}}}+2 H_{2}, \\
& z_{3}=H_{3}+\sqrt{\frac{N_{1}}{H_{1}^{2}}}-2 H_{2}, \quad z_{4}=H_{3}+\sqrt{\frac{N_{2}}{H_{1}^{2}}}+2 H_{2},
\end{aligned}
$$

with

$$
\begin{aligned}
H_{2}= & \sqrt{-\frac{a^{2} a_{01}^{2}}{H_{1}^{2}}}, \quad H_{3}=\frac{a_{01}\left(4 a_{20}-2 b_{11}\right)}{a^{2}+\left(b_{11}-2 a_{20}\right)^{2}}, \\
N_{1}= & a^{4}+2 a^{2}\left(-2 a_{01}^{2}+4 a_{01} H_{2}\left(b_{11}-2 a_{20}\right)+\left(b_{11}-2 a_{20}\right)^{2}\right) \\
& +\left(b_{11}-2 a_{20}\right)^{2}\left(4 a_{01}\left(a_{01}+2 H_{2}\left(b_{11}-2 a_{20}\right)\right)+\left(b_{11}-2 a_{20}\right)^{2}\right), \\
N_{2}= & a^{4}+2 a^{2}\left(\left(b_{11}-2 a_{20}\right)^{2}-2 a_{01}\left(a_{01}+2 H_{2}\left(b_{11}-2 a_{20}\right)\right)\right) \\
& +\left(b_{11}-2 a_{20}\right)^{2}\left(4 a_{01}\left(a_{01}+2 H_{2}\left(2 a_{20}-b_{11}\right)\right)+\left(b_{11}-2 a_{20}\right)^{2}\right) .
\end{aligned}
$$

Thus the differential Eq. (9) with $\varepsilon=0$ can be rewritten into the form

$$
\frac{d r}{d \theta}=r \frac{1}{H_{1}}\left(\frac{\alpha \cos \theta+\beta \sin \theta \cos \theta}{A_{0}+A_{1} \sin \theta+\sin ^{2} \theta}+\frac{\gamma \cos \theta+\delta \sin \theta \cos \theta}{B_{0}+B_{1} \sin \theta+\sin ^{2} \theta}\right),
$$

where $A_{0}=z_{1} z_{2}, A_{1}=-z_{1}-z_{2}, B_{0}=z_{3} z_{4}$ and $B_{1}=-z_{3}-z_{4}$ and the parameters $\alpha, \beta, \gamma$ and $\delta$ are the solutions of the system

$$
\begin{aligned}
& \alpha B_{0}+A_{0} \gamma+8 a_{01} a_{20}=0, \\
& \beta B_{0}+\alpha B_{1}+A_{1} \gamma+A_{0} \delta-a^{2}+8 a_{01}^{2}-4 a_{20}^{2}+4 a_{20} b_{11}-b_{11}^{2}=0, \\
& \beta B_{1}+\alpha+\gamma+A_{1} \delta-8 a_{01} a_{20}+8 a_{01} b_{11}=0, \\
& \beta+\delta+a^{2}+4 a_{20}^{2}-4 a_{20} b_{11}+b_{11}^{2}=0 .
\end{aligned}
$$

The solution of the differential Eq. (10) is

$$
r\left(\theta, r_{0}\right)=k(\theta) r_{0}=r_{0} e^{k_{1}(\theta)} k_{2}(\theta),
$$

where

$$
k_{1}(\theta)=\frac{1}{H_{1}}\left(\frac{\left(2 \alpha-A_{1} \beta\right)\left(\arctan \frac{A_{1}+2 \sin \theta}{\sqrt{R}}-\arctan \frac{A_{1}}{\sqrt{R}}\right)}{\sqrt{R}}+\frac{\left(2 \gamma-B_{1} \delta\right)\left(\arctan \frac{B_{1}+2 \sin \theta}{\sqrt{S}}-\arctan \frac{B_{1}}{\sqrt{S}}\right)}{\sqrt{S}}\right),
$$




$$
\begin{aligned}
k_{2}(\theta) & =A_{0}{ }^{-\frac{\beta}{2 H_{1}}} B_{0}^{-\frac{\delta}{2 H_{1}}}\left(A_{0}+\sin \theta\left(A_{1}+\sin \theta\right)\right)^{\frac{\beta}{2 H_{1}}}\left(B_{0}+\sin \theta\left(B_{1}+\sin \theta\right)\right)^{\frac{\delta}{2 H_{1}}}, \\
S & =4 B_{0}-B_{1}^{2}, \\
R & =4 A_{0}-A_{1}^{2} .
\end{aligned}
$$

Solving the variational Eq. (7) for our differential Eq. (9) we get that the fundamental matrix is $M(\theta)=k(\theta)$. Note that $M(\theta)$ does not depend on $r_{0}$. Using the polynomials $p$ and $q$ given in (3) and system (2) we have that the integrant of the integral (8) for the differential Eq. (10) is

$$
\begin{aligned}
M^{-1}(\theta) F_{1}\left(\theta, r\left(\theta, r_{0}\right)\right) & =\sum_{i=0}^{7} \frac{W(\theta) f_{i}(\theta)}{g_{1}(\theta)^{2} M(\theta)} r\left(\theta, r_{0}\right)^{i-2} \\
& =\sum_{i=0}^{n} r_{0}^{i-2} \frac{W(\theta) f_{i}(\theta)\left(e^{k_{1}(\theta)} k_{2}(\theta)\right)^{i-2}}{g_{1}(\theta)^{2} M(\theta)} \\
& =\sum_{i=0}^{7} r_{0}^{i-2} A_{i}(\theta),
\end{aligned}
$$

where

$$
\begin{aligned}
f_{0}(\theta)= & 8 a_{01}^{2} d_{00} \sin \theta+8 a_{01} a_{20} d_{00} \cos ^{2} \theta, \\
f_{1}(\theta)= & \cos ^{3} \theta\left(a^{2} c_{00}+8 a_{01} a_{20} d_{10}+4 a_{20}^{2} c_{00}-4 a_{20} b_{11} c_{00}+b_{11}^{2} c_{00}\right) \\
& +8 a_{01} \sin \theta \cos \theta\left(a_{01} d_{10}-b_{11} c_{00}\right), \\
f_{2}(\theta)= & \cos ^{4} \theta\left(a^{2} c_{10}+8 a_{01} a_{20} d_{20}+4 a_{20}^{2} c_{10}-4 a_{20} b_{11} c_{10}+b_{11}^{2} c_{10}\right) \\
& +8 a_{01}^{2} d_{01} \sin ^{2} \theta+8 a_{01} \sin \theta \cos ^{2} \theta\left(a_{01} d_{20}+a_{20} d_{01}-b_{11} c_{10}\right), \\
f_{3}(\theta)= & \sin \theta \cos ^{3} \theta\left(a^{2} c_{01}+8 a_{01}^{2} d_{30}+8 a_{01} a_{20} d_{11}-8 a_{01} b_{11} c_{20}+4 a_{20}^{2} c_{01}\right. \\
& \left.-4 a_{20} b_{11} c_{01}+b_{11}^{2} c_{01}\right)+\cos ^{5} \theta\left(a^{2} c_{20}+8 a_{01} a_{20} d_{30}+4 a_{20}^{2} c_{20}\right. \\
& \left.-4 a_{20} b_{11} c_{20}+b_{11}^{2} c_{20}\right)+8 a_{01} \sin ^{2} \theta \cos \theta\left(a_{01} d_{11}-b_{11} c_{01}\right), \\
f_{4}(\theta)= & \sin \theta \cos ^{4} \theta\left(a^{2} c_{11}+8 a_{01} a_{20} d_{21}-8 a_{01} b_{11} c_{30}+4 a_{20}^{2} c_{11}\right. \\
& \left.-4 a_{20} b_{11} c_{11}+b_{11}^{2} c_{11}\right)+c_{30} \cos ^{6} \theta\left(a^{2}+4 a_{20}^{2}-4 a_{20} b_{11}+b_{11}^{2}\right) \\
& +8 a_{01}^{2} d_{02} \sin ^{3} \theta+8 a_{01} \sin ^{2} \theta \cos ^{2} \theta\left(a_{01} d_{21}+a_{20} d_{02}-b_{11} c_{11}\right), \\
f_{5}(\theta)= & \sin { }^{2} \theta \cos ^{3} \theta\left(a^{2} c_{02}+8 a_{01} a_{20} d_{12}-8 a_{01} b_{11} c_{21}+4 a_{20}^{2} c_{02}\right. \\
& \left.-4 a_{20} b_{11} c_{02}+b_{11}^{2} c_{02}\right)+c_{21} \sin \theta \cos ^{5} \theta\left(a^{2}+4 a_{20}^{2}-4 a_{20} b_{11}+b_{11}^{2}\right) \\
& +8 a_{01} \sin ^{3} \theta \cos ^{2} \theta\left(a_{01} d_{12}-b_{11} c_{02}\right), \\
f_{6}(\theta)= & c_{12} \sin ^{2} \theta \cos ^{4} \theta\left(a^{2}+4 a_{20}^{2}-4 a_{20} b_{11}+b_{11}^{2}\right)+8 a_{01}^{2} d_{03} \sin ^{4} \theta \\
& -8 a_{01} \sin ^{3} \theta \cos ^{2} \theta\left(b_{11} c_{12}-a_{20} d_{03}\right), \\
f_{7}(\theta)= & c_{03} \sin ^{3} \theta \cos ^{3} \theta\left(a^{2}+4 a_{20}^{2}-4 a_{20} b_{11}+b_{11}^{2}\right) \\
& -8 a_{01} b_{11} c_{03} \sin ^{4} \theta \cos ^{2} \theta, \\
W(\theta)= & -8 a_{01}\left(2 \sin ^{2} \theta+\cos ^{2} \theta\right) .
\end{aligned}
$$

Computing the integral (8) we obtain

$$
\mathcal{F}\left(r_{0}\right)=\frac{1}{2 \pi} \int_{0}^{2 \pi} M^{-1}(\theta) F_{1}\left(\theta, r\left(\theta, r_{0}\right)\right) d \theta=\frac{1}{2 \pi} \sum_{i=0}^{n} r_{0}^{i-2} \int_{0}^{2 \pi} A_{i}(\theta) d \theta
$$

where the function $A_{i}(\theta)$ are defined in the statement of Theorem 1.

If $i$ is odd then it is easy to check that $f_{i}(\theta)=-f_{i}(\theta+\pi / 2)$, for $i=0, \ldots, 7$, and $\theta \in[0, \pi / 2] \cup[\pi, 3 \pi / 2]$. Since that $k_{1}(\theta)=$ $k_{1}(\theta+\pi / 2), k_{2}(\theta)=k_{2}(\theta+\pi / 2), M(\theta)=M(\theta+\pi / 2)$ and $W(\theta)=W(\theta+\pi / 2)$, for $\theta \in[0, \pi / 2] \cup[\pi, 3 \pi / 2]$, we easily obtain

$$
\begin{aligned}
\int_{\frac{\pi}{2}}^{\pi} A_{i}(\theta) d \theta & =\int_{\frac{\pi}{2}}^{\pi} \frac{W(\theta) f_{i}(\theta)\left(e^{k_{1}(\theta)} k_{2}(\theta)\right)^{i-2}}{g_{1}(\theta)^{2} M(\theta)} d \theta \\
& =\int_{0}^{\frac{\pi}{2}} \frac{W(\theta+\pi / 2) f_{i}(\theta+\pi / 2)\left(e^{k_{1}(\theta+\pi / 2)} k_{2}(\theta+\pi / 2)\right)^{i-2}}{g_{1}(\theta+\pi / 2)^{2} M(\theta+\pi / 2)} d \theta \\
& =\int_{0}^{\frac{\pi}{2}}-\frac{W(\theta) f_{i}(\theta)\left(e^{k_{1}(\theta)} k_{2}(\theta)\right)^{i-2}}{g_{1}(\theta)^{2} M(\theta)} d \theta
\end{aligned}
$$




$$
\begin{aligned}
& =-\int_{0}^{\frac{\pi}{2}} A_{i}(\theta) d \theta \\
\int_{\frac{3 \pi}{2}}^{2 \pi} A_{i}(\theta) d \theta & =\int_{\frac{3 \pi}{2}}^{2 \pi} \frac{W(\theta) f_{i}(\theta)\left(e^{k_{1}(\theta)} k_{2}(\theta)\right)^{i-2}}{g_{1}(\theta)^{2} M(\theta)} d \theta \\
& =\int_{\pi}^{\frac{3 \pi}{2}} \frac{W(\theta+\pi / 2) f_{i}(\theta+\pi / 2)\left(e^{k_{1}(\theta+\pi / 2)} k_{2}(\theta+\pi / 2)\right)^{i-2}}{g_{1}(\theta+\pi / 2)^{2} M(\theta+\pi / 2)} d \theta \\
& =\int_{\pi}^{\frac{3 \pi}{2}}-\frac{W(\theta) f_{i}(\theta)\left(e^{k_{1}(\theta)} k_{2}(\theta)\right)^{i-2}}{g_{1}(\theta)^{2} M(\theta)} d \theta \\
& =-\int_{\pi}^{\frac{3 \pi}{2}} A_{i}(\theta) d \theta .
\end{aligned}
$$

Therefore for $i$ odd we have

$$
\int_{0}^{2 \pi} A_{i}(\theta) d \theta=0
$$

Analogously if $i$ is even then a function $g \in\left\{k_{1}, k_{2}, M, W, f_{i}\right.$, for $\left.i=0, \ldots, 7\right\}$ satisfies that $g(\theta)=g(\theta+\pi / 2)$ if $\theta \in[0, \pi / 2] \cup[\pi$, $3 \pi / 2]$. Thus we easily can check that

$$
\int_{0}^{\frac{\pi}{2}} A_{i}(\theta) d \theta=\int_{\frac{\pi}{2}}^{\pi} A_{i}(\theta) d \theta \quad \text { and } \quad \int_{\pi}^{\frac{3 \pi}{2}} A_{i}(\theta) d \theta=\int_{\frac{3 \pi}{2}}^{2 \pi} A_{i}(\theta) d \theta .
$$

So we have

$$
\int_{0}^{2 \pi} A_{i}(\theta) d \theta=2 \int_{\frac{\pi}{2}}^{\frac{3 \pi}{2}} A_{i}(\theta) d \theta \neq 0 .
$$

Therefore the function $\mathcal{F}$ defined in (8) can be written as

$$
\mathcal{F}\left(r_{0}\right)=\frac{1}{2 \pi} \sum_{k=0}^{3} r_{0}^{2 k-2} \int_{0}^{2 \pi} A_{2 k}(\theta) d \theta .
$$

Note that the coefficients $A_{2 k}(\theta)$ in (11) are linearly independent for $k=0, \ldots, 3$. Thus by the generalized Descartes Theorem, the averaged function $\mathcal{F}$ has at most 3 positive simple zeros which provide limit cycles of system (3). Hence statement (a) of Theorem 1 is proved.

\section{Proof of statement (b) of Theorem 1}

In this section we present examples that illustrate statement (b) of Theorem 1 for $l=0,1,2,3$.

Consider the cubic polynomial differential system with a center at the origin

$$
\dot{x}=x^{2}+y, \quad \dot{y}=-\frac{x^{3}}{4}+3 x y,
$$

with the perturbation

$$
\dot{x}=x^{2}+y, \quad \dot{y}=-\frac{x^{3}}{4}+3 x y+\varepsilon\left(d_{00}+d_{20} x^{2}+d_{21} x^{2} y+d_{03} y^{3}\right) .
$$

Writing system (12) in the coordinates $x=r \cos \theta$ and $y=r^{2} \sin \theta$, and taking the quotient $\dot{r} / \dot{\theta}$ we obtain the following system in the standard form of Theorem 2 for applying the averaging theory

$$
\frac{d r}{d \theta}=F_{0}(r, \theta)+\varepsilon F_{1}(r, \theta)+\mathcal{O}\left(\varepsilon^{2}\right),
$$

where

$$
\begin{aligned}
F_{0}(r, \theta) & =\frac{r \cos \theta(-15 \sin \theta+\sin (3 \theta)+16 \cos (2 \theta)-32)}{4\left(8 \sin ^{2} \theta+\cos ^{4} \theta-4 \sin \theta \cos ^{2} \theta\right)}, \\
F_{1}(r, \theta) & =-\frac{16 H(\theta)\left(d_{00}+d_{03} r^{6} \sin ^{3} \theta+d_{20} r^{2} \cos ^{2} \theta+d_{21} r^{4} \sin \theta \cos ^{2} \theta\right)}{r^{2}\left(8 \sin ^{2} \theta+\cos ^{4} \theta-4 \sin \theta \cos ^{2} \theta\right)^{2}}, \\
H(\theta) & =\left(\cos ^{2} \theta+\sin \theta\right)\left(\cos ^{2} \theta+2 \sin ^{2} \theta\right) .
\end{aligned}
$$


Thus for system (13) we have

$$
k(\theta)=M(\theta)=\frac{2^{3 / 4} \exp \left(-\frac{5}{2} \arctan \frac{4 \sin \theta}{-4 \sin \theta+\cos (2 \theta)+1}\right)}{\sqrt[4]{-8 \sin \theta-8 \sin (3 \theta)-28 \cos (2 \theta)+\cos (4 \theta)+35}},
$$

and the integrant of the integral (8) of system (13) is

$$
\frac{A(\theta)+B(\theta) r_{0}^{2}+C(\theta) r_{0}^{4}+D(\theta) r_{0}^{6}}{r_{0}^{2}}
$$

where

$$
\begin{aligned}
& A(\theta)=-\frac{2^{31 / 4} d_{00} H(\theta) \exp \left(\frac{15}{2} \arctan \frac{4 \sin \theta}{-4 \sin \theta+\cos (2 \theta)+1}\right)}{(-8 \sin \theta-8 \sin (3 \theta)-28 \cos (2 \theta)+\cos (4 \theta)+35)^{5 / 4}}, \\
& B(\theta)=-\frac{2^{37 / 4} d_{20} H(\theta) \cos ^{2} \theta \exp \left(\frac{5}{2} \arctan \frac{4 \sin \theta}{-4 \sin \theta+\cos (2 \theta)+1}\right)}{(-8 \sin \theta-8 \sin (3 \theta)-28 \cos (2 \theta)+\cos (4 \theta)+35)^{7 / 4}}, \\
& C(\theta)=-\frac{2^{43 / 4} d_{21} H(\theta) \sin \theta \cos ^{2} \theta \exp \left(-\frac{5}{2} \arctan \frac{4 \sin \theta}{-4 \sin \theta+\cos (2 \theta)+1}\right)}{(-8 \sin \theta-8 \sin (3 \theta)-28 \cos (2 \theta)+\cos (4 \theta)+35)^{9 / 4}}, \\
& D(\theta)=-\frac{2^{49 / 4} d_{03} H(\theta) \sin ^{3} \theta \exp \left(-\frac{15}{2} \arctan \frac{4 \sin \theta}{-4 \sin \theta+\cos (2 \theta)+1}\right)}{(-8 \sin \theta-8 \sin (3 \theta)-28 \cos (2 \theta)+\cos (4 \theta)+35)^{11 / 4}}
\end{aligned}
$$

Computing numerically the integral (8) for system (13) we obtain

$$
\mathcal{F}\left(r_{0}\right)=\frac{1}{r_{0}^{2}}\left(-427527.30772 . . d_{00}-590.65521 . . d_{20} r_{0}^{2}-133.80248 . . d_{21} r_{0}^{4}-48202.93168 . . d_{03} r_{0}^{6}\right) .
$$

Taking

$$
\begin{aligned}
& d_{00}=\frac{-36}{427527.30772 . .}, \quad d_{20}=\frac{-49}{590.65521 . .}, \quad d_{21}=\frac{-14}{133.80248 . .}, \\
& d_{03}=\frac{-1}{48202.93168 . .},
\end{aligned}
$$

the function $\mathcal{F}$ becomes

$$
\mathcal{F}\left(r_{0}\right)=\frac{36+49 r_{0}^{2}+14 r_{0}^{4}+r_{0}^{6}}{r_{0}^{2}}
$$

We can easily check that $\mathcal{F}$ does not have positive simple zeros and by Theorem 2 the perturbed system (12) does not have limit cycles with $\varepsilon \neq 0$ sufficiently small. So statement (b) is proved for $l=0$.

Get

$$
\begin{aligned}
& d_{00}=\frac{36}{427527.30772 . .}, \quad d_{20}=\frac{41}{590.65521 . .}, \quad d_{21}=\frac{4}{133.80248 . .}, \\
& d_{03}=\frac{-1}{48202.93168 . .}
\end{aligned}
$$

the function $\mathcal{F}$ is now given by

$$
\mathcal{F}\left(r_{0}\right)=\frac{-36-41_{0}^{2}-4 r_{0}^{4}+r_{0}^{6}}{r_{0}^{2}} .
$$

Thus we have that $\mathcal{F}$ has a unique positive simple zero given by $r_{0,1}=3$ which by Theorem 2 , provide 1 limit cycle of the perturbed system (12) with $\varepsilon \neq 0$ sufficiently small. This shows statement (b) for $l=1$.

Taking

$$
\begin{aligned}
& d_{00}=\frac{-36}{427527.30772 . .}, \quad d_{20}=\frac{-23}{590.65521 . .}, \quad d_{21}=\frac{12}{133.80248 . .}, \\
& d_{03}=\frac{-1}{48202.93168 . .}
\end{aligned}
$$

the function $\mathcal{F}$ turns into

$$
\mathcal{F}\left(r_{0}\right)=\frac{36+23 r_{0}^{2}-12 r_{0}^{4}+r_{0}^{6}}{r_{0}^{2}} .
$$

We can check that $\mathcal{F}$ has exactly 2 positive simple zeros given by $r_{0,1}=2$ and $r_{0,2}=3$ which by Theorem 2 , provide 2 limit cycles of the perturbed system (12) with $\varepsilon \neq 0$ sufficiently small. Therefore statement (b) is proved for $l=2$. 
Finally, taking

$$
\begin{aligned}
& d_{00}=\frac{36}{427527.30772 . .}, \quad d_{20}=\frac{-49}{590.65521 . .}, \quad d_{21}=\frac{14}{133.80248 . .}, \\
& d_{03}=\frac{-1}{48202.93168 . .}
\end{aligned}
$$

the function $\mathcal{F}$ becomes

$$
\mathcal{F}\left(r_{0}\right)=\frac{-36+49_{0}^{2}-14 r_{0}^{4}+r_{0}^{6}}{r_{0}^{2}} .
$$

Thus it is easy to check that $\mathcal{F}$ has exactly 3 positive simple zeros given by $r_{0,1}=1, r_{0,2}=2$ and $r_{0,3}=3$ which by Theorem 2 , provide 3 limit cycles of the perturbed system (12) with $\varepsilon \neq 0$ sufficiently small. Hence statement (b) is proved.

\section{Acknowledgments}

The first author is partially supported by a MINECO/FEDER grant MTM2008-03437, MINECO grant MTM2013-40998-P, an AGAUR grant number 2014SGR-568, an ICREA Academia, the grants FP7-PEOPLE-2012-IRSES 318999 and 316338, the MINECO/FEDER grant UNAB13-4E-1604, and a CAPES grant number 88881.030454/2013-01 from the program CSF-PVE. The second author is partially supported by the joint projects FP7-PEOPLE-2012-IRSES numbers 316338, CAPES grant number 88881.030454/2013-01 from the program CSF-PVE and CNPq grant "Projeto Universal 472796/2013-5". The second author is supported by CAPES/GDU-7500/13-0.

\section{References}

[1] A.A. Andronov, E.A. Leontovich, I.I. Gordon, A.G. Maǐer, Theory of Bifurcations of Dynamic Systems on a Plane, Halsted Press [A division of John Wiley \& Sons], New York-Toronto, Ont., 1973. Translated from the Russian.

[2] I.S. Berezin, N.P. Zhidkov, Computing Methods, II, Pergamon Press, Oxford, 1964.

[3] T.R. Blows, L.M. Perko, Bifurcation of limit cycles from centers and separatrix cycles of planar analytic systems, SIAM Rev. 36 (1994) 341-376.

[4] A. Buică, J.P. Françoise, J. Llibre, Periodic solutions of nonlinear periodic differential systems with a small parameter, Commun. Pure Appl. Anal. 6 (2007) $103-111$.

[5] A. Buică, J. Llibre, Averaging methods for finding periodic orbits via Brouwer degree, Bull. Sci. Math. 128 (2004) 7-22.

[6] C. Christopher, C. Li, Limit Cycles in Differential Equations, Birkhauser, Boston, 2007.

[7] F. Dumortier, J. Llibre, J.C. Artés, Qualitative Theory of Planar Differential Systems, Universitext, Springer-Verlag, Berlin, 2006.

[8] J. Giné, Higher order limit cycle bifurcations from non-degenerate centers, Appl. Math. Comput. 218 (2012) 8853-8860.

[9] D. Hilbert, Mathematische Probleme, in: Lecture, Second Internat. Congr. Math., Paris, 1900, in: Nachr. Ges. Wiss. Göttingen Math. Phys. KL., 1900, pp. 253-297, English transl.: Bull. Amer. Math. Soc. 8 (1902) 437-479.

[10] J. Llibre, B.D. Lopes, J.R. de Moraes, Limit cycles of cubic polynomial differential systems with rational first integrals of degree 2, Appl. Math. Comput. 250 (2015) 887-907.

[11] J. Llibre, C. Pessoa, On the centers of the weight-homogeneous polynomial vector fields on the plane, J. Math. Anal. Appl. 359 (2009) 722-730.

[12] J. Llibre, C. Valls, Classification of the centers, of their cyclicity and isochronicity for two classes of generalized quintic polynomial differential systems, NoDEA Nonlinear Differ. Equ. Appl. 16 (2009) 657-679.

[13] J.A. Sanders, F. Verhulst, J. Murdock, Averaging methods in nonlinear dynamical systems, Applied Mathematical Sciences, 59 , Springer, New York, 2007.

[14] J. Shi, J. Li, Bifurcations of limit cycles in a $Z_{6}$-equivariant planar vector field of degree 7, Appl. Math. Comput. 244 (2014) 191-200.

[15] S. Smale, Mathematical problems for next century, Math. Intell. 20 (1998) 7-15. 